\title{
Building a Software System Using a Spiral Model to Evaluate the Overall Risk of Projects Using Fuzzy Number Theory
}

Jamal Salah Al-Din Sayed Majeed

Ro'a Amer Hussain

College of Computer Science and Mathematics

University of Mosul, Mosul, Iraq

\section{Received on: 18/10/2012}

Accepted on: 30/01/2013

\section{ABSTRACT}

This research aims presented a practical application for the process of assessing the overall risk of the projects following the risk management in software engineering steps, the life cycle is the most famous in this area is a (Spiral Model), this model represented by the core of the process to building a system based on customers information taken from them in the form of hearings conversations. This research depended on EA tool (Enterprise Architect) that building by a company (Sparex Systems) for represent the processes of stages to analysis because it is the basis currently used in the world and the most prevalent and most recently the base idea for this work is found the rate of aggregative risk in project by used fuzzy model for fuzzy number by follow Lee's algorithm. This system has been applied a practical in bank / public administration / northern region of the data / analysis and risk assessment of bank credit / short-term and long-term.

Keywords: Spiral Model, Enterprise Architect, Fuzzy Number, Lee Algorithm,

$$
\begin{aligned}
& \text { بناء نظام برمجي بإتباع النموذج اللولبي لتقييم الخطر الإجمالي للمشاريع باستخدام نظرية التضبيب الرقيمي } \\
& \text { جمال صلاح الاين سيا مجيد } \\
& \text { كلية علوم الحاسوب والرياضيات، جامعة الدوصل }
\end{aligned}
$$$$
\text { تاريخ قبول البحث: 2013/01/30 }
$$$$
\text { تاريخ استلام البحث: 2012/10/18 }
$$

\section{الملخص}

يهدف البحث إلى تقديم تطبيق عملي لعملية تقييم المخاطر الكلية للمشاريع بإتباع خطوات إدارة المخاطر

في هندسة البرمجيات، دورة حياة التي تعد الأثهر في هذا المجال وهو النموذج الحلزوني (Spiral Model) الذي يشكل الأساس في عملية بناء النظام الخاص ببحثنا بالاعتماد على معلومات الزبون التي تؤخذ منه على شكل جلسات حوارية. تم الاعتماد في هذا البحث على الأداة Enterprise Architect) EA) المصنع من قبل شركة

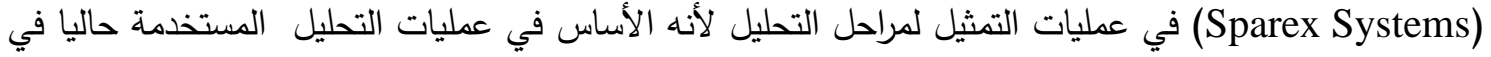
Rate of ) العالم وأكثرها انتشارا وأحدثها. تركز الفكرة الأساسية لهذا العمل على إيجاد النسبة الكلية للخطر الكلي (Fuzzy Number) في المشروع باستخدام النموذج الضبابي الخاص بالتضبيب الرقمي (aggregative Risk المتبع في خوارزمية لي(Lee Algorithm). تم تطبيق هذا النظام بثكل عملي على بنك الرشيد / الإدارة العامة / المنطقة الثمالية للبيانات الخاصة / تحليل وتقييم مخاطر البنك الائتمانية / قصيرة وطويلة الأجل.

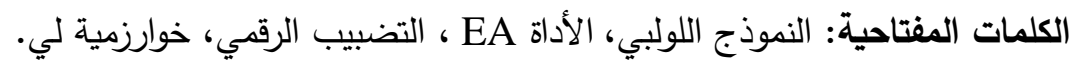

أصبحت صناعة البرمجيات لمجتمعاتتا المعاصرة ترتبط ارتباطاً وثيقاً بمستلزمات حياته ونموه وازدهاره،

وهو منهج يهتم بدورة تطوير وإنشاء البرمجيات والتي تتضمن كل من تجميع وتحليل المتطلبات، التصميم والبناء، الاختبار والصيانة، وإدارة اقتصاديات هندسة البرمجيات[1][2]، يطلق على هذه المراحل اسم دورة حياة النظام 
البرمجي (Software Life cycle) التي قد يبدو بعضها ليس له علاقة بالبرمجة وهناك الكثير من التصورات

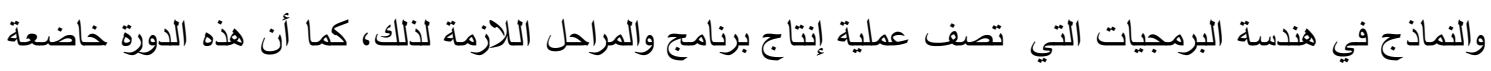
للتطوير دائماً.][3]. 2

إن الأنموذج المعتمد في بناء النظام هو الأنموذج الحلزوني-ويطلق عليه أيضاً في بعض الأحيان بالأنموذج اللولبي- هذا الأنموذج معقد ومتقدم اقترحه الباحث باري بوهيم 1968م (Barry Boehm)، وهو

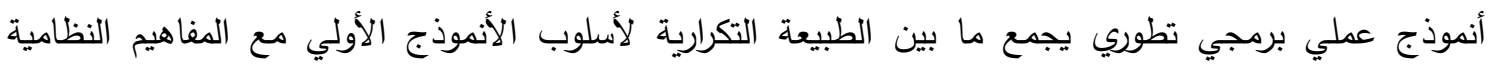

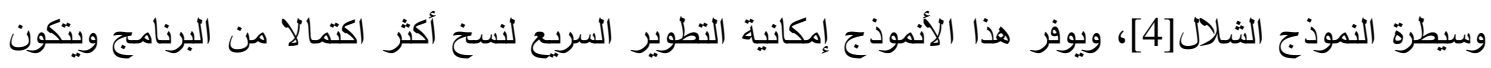
من مراحل عديدة: 1. المرحلة الأولى: في هذه المرحلة يجب على فريق العمل أن يضع في حسبانه كل مواطِن الأخطار المحدقة

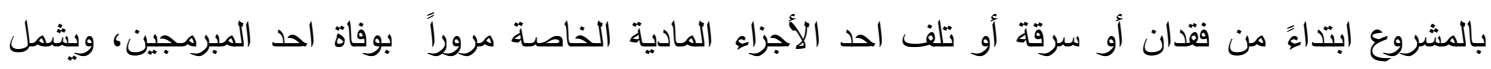

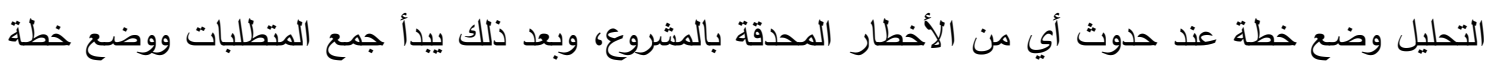
ووضع الخطة البديلة. بهذا يكون أنموذج1 (prototype1) المبدئي للنظام أو المشروع جاهزاً.

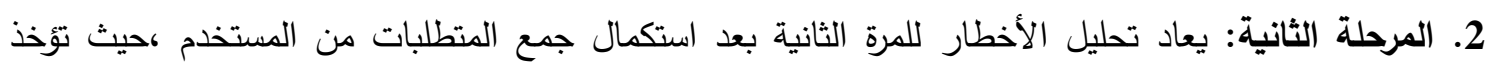

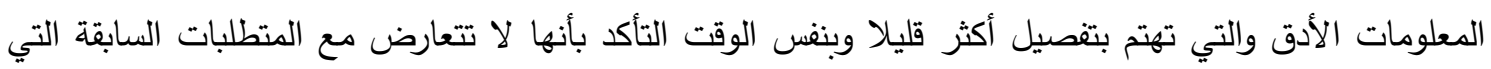
أرادها الزبون وبذلك نحصل على الأنموذج2 (prototype2) وهذا الأنموذج يعتمد عليه في إعطاء كافة الأخطار المحدقة بتفاصيل للنظام. 3. المرحلة الثالثة: تحليل الأخطار للمرة الثالثة، وفي هذه المرحلة أيضا يبدأ عمل النماذج للنظام بأكمله للمقارنة مع ما أراده الزبون وعمل خطة التجميع لكل النماذج.

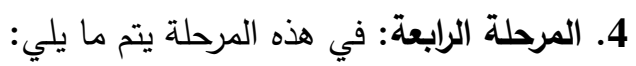

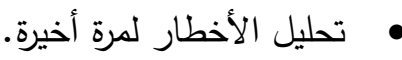

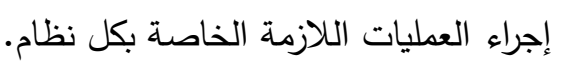
عمل التصميم التنصيلي الدقيق. كتابة الثفرة. التجميع.

الاختبار الجزئي والكلي والذي يسمى باختبار القبول (Acceptance test). تسيلم الأنموذج العرض الرابع (prototype 4) وهذا الأنموذج يسمى الامية التثغيلية ويجري عليه اختبار الموافقة أو القبول من قبل المستخدم. هذا الأنموذج طويل ومعقد ولكنه يوفر تكاليف باهظة في حالة حصول أخطاء بسبب وجود عملية التقصي عن الأخطار في كل خطوة وبذلك فان الخطأ لا يتعدى المرحلة اللاحقة [5]. يجب الانتباه إلى المرحلة الأولى بشكل خاص بسبب كونها العمود المهم لمراحل التطوير ويعتمد عليها في

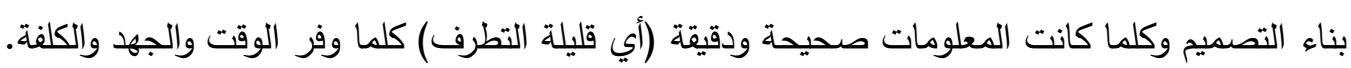

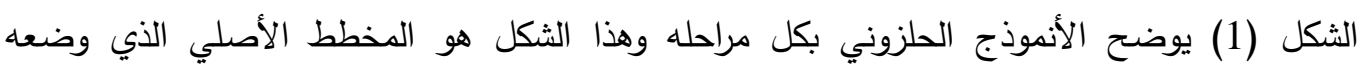
Boehm 
الخاص لها لكن تبقى الخطوات المذكورة أعلاه هي الخطوات الأساس ويكن أن يحصل التغيير في عدد تكرار

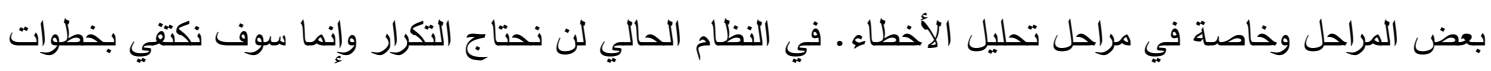

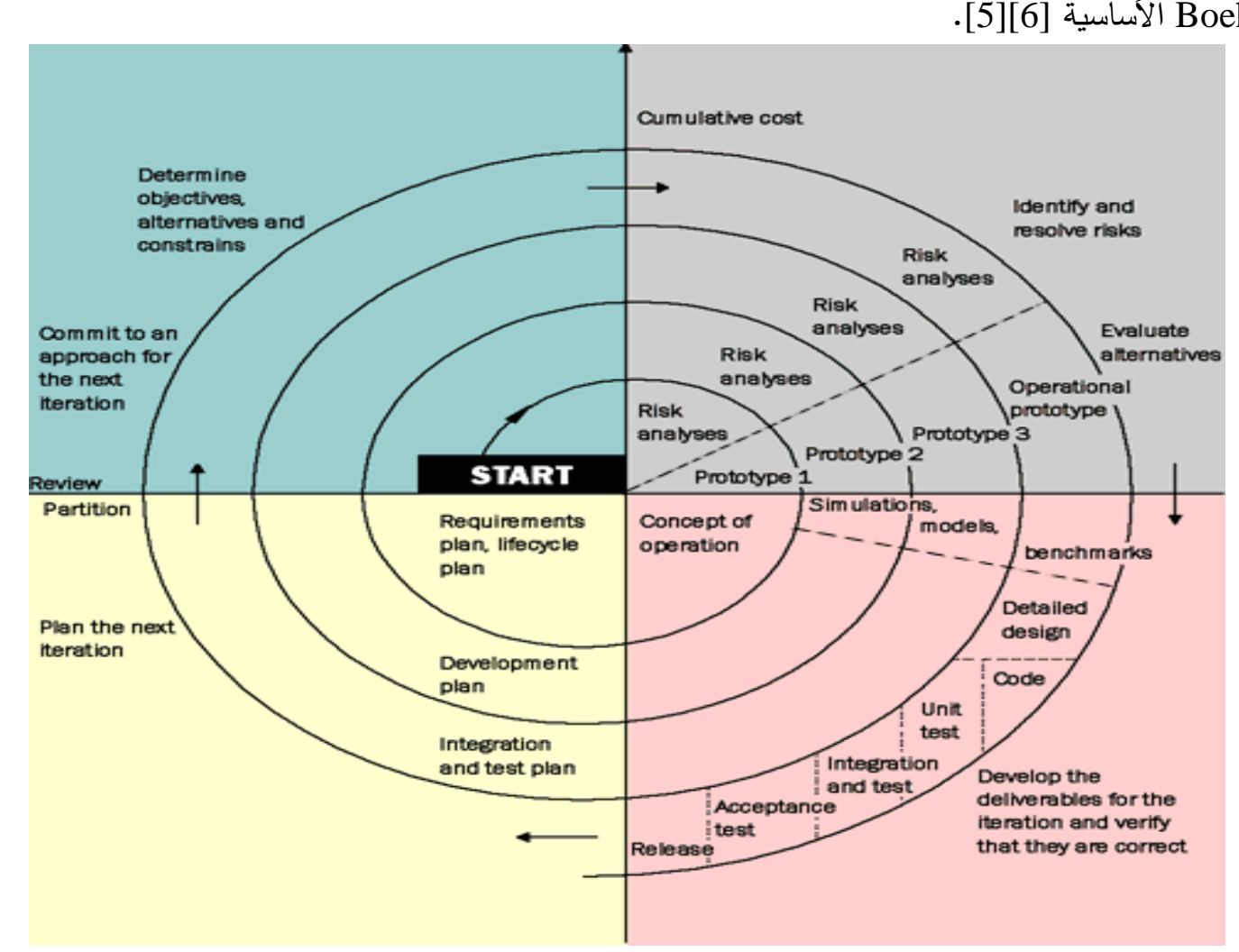

شكل (1). يوضح أنموذج الحلزوني (Spiral Model)

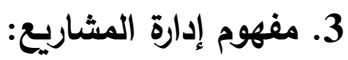

إن إدارة المشروع هي تطبيق المعارف، المهارات، الأدوات والتتنيات على نشاطات المشروع، لتحقيق

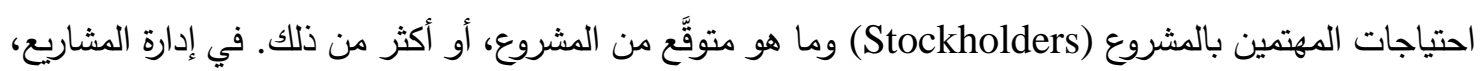
يقوم الرئيس المؤَّت للمشروع (Transient Project Leader) والذي يسمى مدير المشروع (Sroject leader) بتوجيه الإدارة، من خلال الاستفادة الكاملة من الموارد المتوفرة بما فيها الموارد البشرية وذلك لتحقيق الغاية من فئن المشروع و الهدف والإنجازات ضمن حدود الكلفة المتوقَّعة.التثبت والتحقق من المتطلبات التي تم تسجليها في كلا من وثيقة تعريف المتطلبات (والتي تقدم للعميل) ووثيقة مواصفات المتطلبات (والتي تقدم للمصدم) للتأكد من فن فن

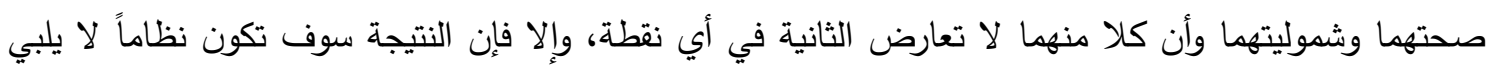

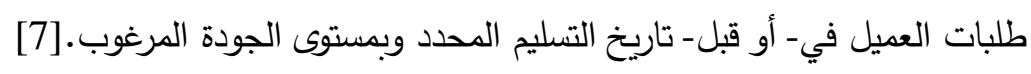

4. إدارة المخاطر:

يعد مفهوم المخاطرة جزءاً لا يتجزأ من النشاط الإنساني مهما كانت طبيعته. وهي في النشاط الاقتصادي

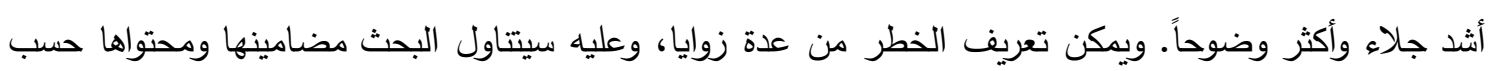

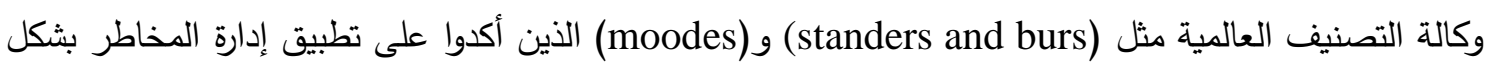
كامل وأنها عامل مؤثر في رفع درجة التصنيف للمخاطر [8][7]. 


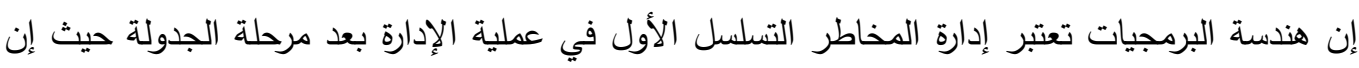

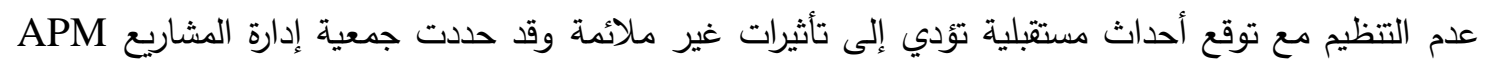

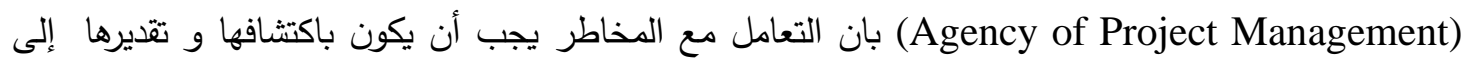

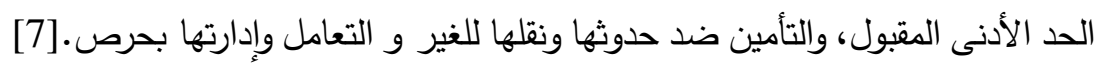

2.4

إن معهد إدارة المشاريع (Project Management Institute) قام بتصنيف استراتيجيات التدخل لاى حدوث المخاطر وهي كما يلي:

1.2.4 إستراتيجيات المخاطر المنفعلة (Reactive Risk Strategies):

تعتمد معظم الفرق البرمجية على الاستراتيجيات المنفعلة لإدارة المخاطرة حيث تراقب الإستراتيجية المنفعلة

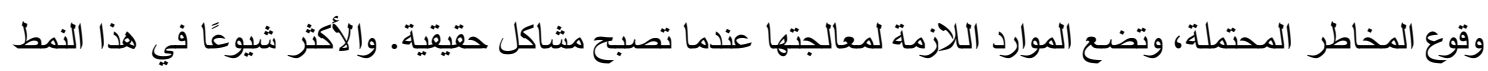

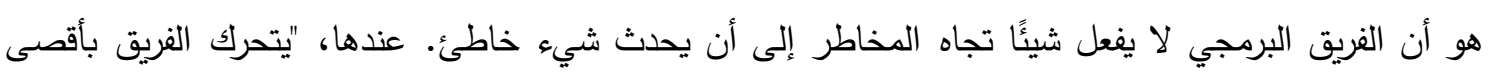

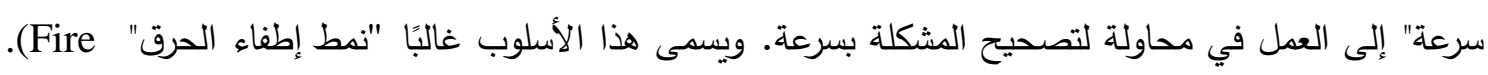
وعندما يخفق ذلك يجري اعتماد أسلوب" إدارة الأزمات (Crisis Managementing Mode)

$$
\text { المشروع في خطر حقيقي[4]. }
$$

2.2.4 إستراتيجيات المخاطر الفاعلة (Proactive Risk Strategies):

الإستراتيجية الأكثر ذكاءاً لإدارة المخاطرة هي الإستراتيجية الفاعلة، والتي تبدأ قبل بدء العمل التقني بكثير .

حيث يجري تحديد المخاطر (Risks Identification) المحتملة وتقدير احتمال وقوعها (Occurrence)

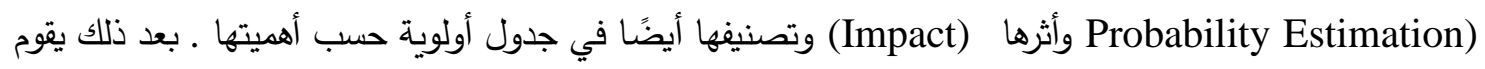
الفريق البرمجي بوضع خطة لإدارة المخاطرة (Risk Plan Management) وخطة الطوارئ ( Contingency) .[4](Plan

3.4

بالرغم من أن هناك العديد من الاقتراحات لتعريف المخاطرة البرمجية (Software Risk) إلا أن هناك اتفاقاً عاماً على أن المخاطرة تتصف دومًا بصفتين: • عدم اليقين (Uncertainty): قد يحصل الحدث المميز للمخاطرة وقد لا يحصل، أي لا يوجد مخاطر

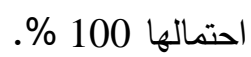
• الخسارة (Loss): إذا أصبحت المخاطرة حقيقية فإن التبعات أو الخسائر الناتجة عن ذلك ستحصل.

4.4

من الضروري عند تحليل المخاطر إعطاء قيمة كمية لمستوى الثك ودرجة الخسارة المتعلقة بكل منها. ولتحقيق ذلك تصنَّف المخاطرة إلى الأصناف التالية: المخاطرة التقنية (Technical Risk): تهدد المخاطر التقنية جودة ودقة وتوقيت البرمجية المطلوب إنتاجها. وعند حصول مخاطرة تقنية، يصبح تحقيق البرمجية صعبًا أو مستحيلا. تحدد المخاطر التقنية المشاكل المحتملة في تهدي 
التصميم، التحقيق، الواجهات، الاختبار، والصيانة. إضافة إلى ذلك هناك عوامل أخرى للمخاطرة في هذا الإطار، كغوض المواصفات (Specification Ambiguity)، الثك التقني (Technical Uncertainty)، التقادم التقني (Technical Forward)، والتقنيات الجديدة. تحصل الدخاطرة التقنية في أغلب الأحيان لأن المسألة أصعب حلا مما تصورنا.

مخاطرة داخلية (Internal Risk): الوقت، الكلفة، النطاق، الجودة، التخطيط، الموارد البشرية، المواد والتجهيزات.

مخاطرة السوق (Market Risk): بناء منتج أو نظام ممتاز لا يرغب فيه أحد وهل سيكون المنتج الجديد مفيدًا وقابلاً للتسويق للآخرين. مخاطرة الجدول الزمني (Schedule Risk): وهي درجة الثك في أن يحاَظظ على الجدول الزمني للمشروع وأن يسلَّم المنتّج في موعده. مخاطرة الدعم (support Risk): وهي درجة الثك في أن تكون البرمجيات سهلة التصحيح والتكييف والتحسين. مخاطرة الأداء (Performance Risk): وهي درجة الثك في أن يتوافق المنتج مع المتطلبات المطلوبة منها، وأن يناسب الاستخدام المخطُّط له.

من المهم ملاحظة أن هذا التصنيف البسيط لن يعمل دومًا، فبعض المخاطر لا يمكن التتبؤ بها سلفًا.[4][5] 5. تعتبر الفكرة الأساسية القائم عليها النظام المصدم هي تقييم المخاطر ( Risk Assessment) للمشاريع دون التطرق لطرق المعالجة والسيطرة (Risk Mitigate \& Monitor) والمراحل الآتية تعرف بشكل مفصل لكل مرحلة من مراحل عملية التقييم[4][9][10]. الثكل (2) يوضح الخطوات الأساسية للنظام المستخدم في التقييم.

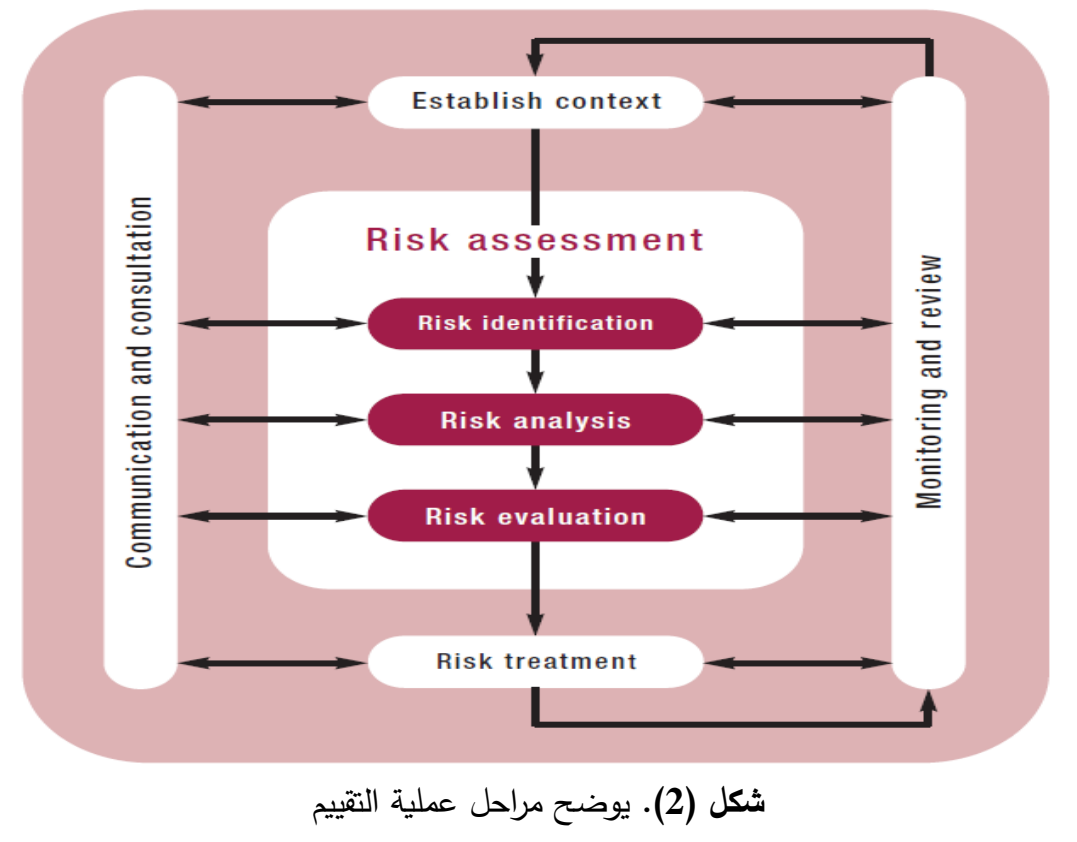


تعريف المخاطرة أو تحديد هوية المخاطرة (Risk Identification) هو إجراء لتوصيف التهديدات التي تعترض خطة المشروع عندما يقوم مدير المشروع بتعيين هوية المخاطر ، فإنه يكون قد خطا الخطوة الأولى باتجاه

$$
\text { تجنبها عند الإمكان والتحكم فيها أيضًا عند الضرورة مديرة }
$$

هناك نوعان متميزان من المخاطر لجميع أصناف أو فئات المخاطر، هخاطر العامة (Generic Risks) والمخاطر الخاصة (Specific Risks) .المخاطر العامة هي تهديد محتمل لكل مشروع برمجي، أما المخاطر

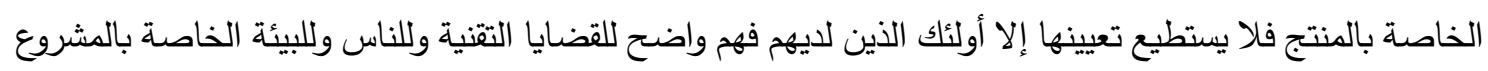
الجاري تتفيذه .يجري فحص خطة المشروع وبيان نطاق البرمجيات بهدف تحديد المخاطر ، ويوضع جواب عن السؤال

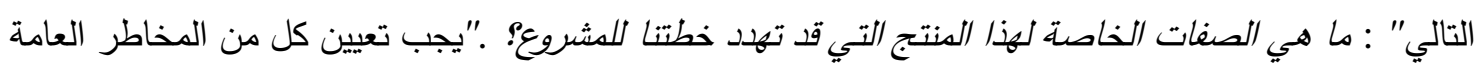
والخاصة بالمنتج بشكل نظامي، إحدى طرائق تحديد المخاطر هي أن نقوم بإنشاء قائمة حصر لعناصر المخاطرة (Risk Item Checklist)._وهو ما ستتناوله الباحثة في تطبيق النظام_ وتعتبر هذه المرحلة من أهم خطوات العمل.10][10]

2.5 تحليل المخاطر :

كل نوع من أنواع الأخطار يتم أخذه وتجرى له عملية توصيف ( description)، هناك عدة طرق لعملية

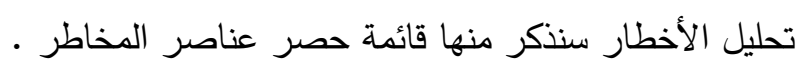

\subsection{5 قائمة حصر عناصر المخاطر :}

إحدى طرائق تحليل المخاطر هي أن نقوم بإنثاء قائمة حصر عناصر المخاطرة يمكن استخدام هذه القائمة

لتحديد المخاطر وتركيز الاهتمام في مجموعة جزئية من المخاطر المعروفة والقابلة للتتبؤ بها في الفئات الجزئية العمومية والتي تختلف من مشروع لآخر وبذلك تختلف قائمة تفقُّ العناصر المتعلقة بكل منها. إن التقييم الذاتي يقوم على إعداد قائمة بالعناصر القابلة للتعرض إلى المخاطر (مثلا: القيادة، العمال، الزبائن، الإستراتيجية والعمليات، الموارد الأخرى، نتائج الثركة) وقياس مدى التغيرات الحاملة للخسائر بواسطة مصفوفة محتواة في

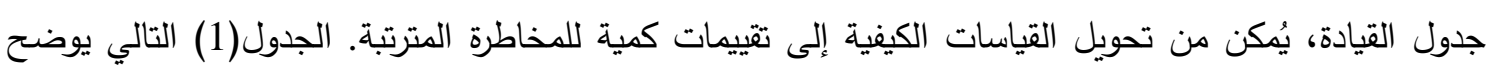

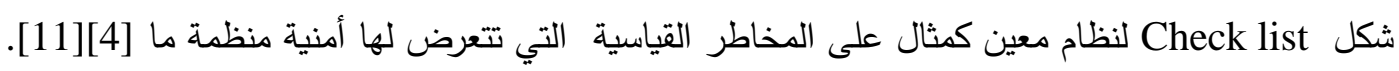
جدول (1). يوضح هيئة قائمة حصر لعناصر الخطر لنظام أمنية منظمة ما.

\begin{tabular}{l|l|} 
External parties & $\begin{array}{l}\text { To maintain the security of the organization's } \\
\text { information and information processing facilities } \\
\text { that are accessed processed, communicated to, or } \\
\text { managed by external parties. }\end{array}$ \\
\hline Identification of risks related to external parties & $\begin{array}{l}\text { The risks to the organization's information and } \\
\text { information processing facilities from business } \\
\text { processes involving external parties shall be } \\
\text { identified and appropriate controls implemented } \\
\text { before granting access. }\end{array}$ \\
\hline $\begin{array}{l}\text { Addressing security when dealing with } \\
\text { customers }\end{array}$ & $\begin{array}{l}\text { All identified security requirements shall be } \\
\text { addressed before giving customers access to the } \\
\text { organization's information or assets. }\end{array}$ \\
\hline
\end{tabular}




\begin{tabular}{|l|l|} 
Addressing security in third party agreements & $\begin{array}{l}\text { Agreements with third parties involving } \\
\text { accessing, processing, communicating or } \\
\text { managing the organization's information or } \\
\text { information processing facilities, or adding } \\
\text { products or services to information processing } \\
\text { facilities shall cover all relevant security } \\
\text { requirements. }\end{array}$ \\
\hline Asset management & $\begin{array}{l}\text { To achieve and maintain appropriate protection of } \\
\text { organizational assets. }\end{array}$ \\
\hline Responsibility for assets & $\begin{array}{l}\text { All assets shall be clearly identified and an } \\
\text { inventory of all important assets drawn up and } \\
\text { maintained. }\end{array}$ \\
\hline Inventory of assets & $\begin{array}{l}\text { All information and assets associated with } \\
\text { information processing facilities shall be owned } \\
\text { by a designated part of the organization. }\end{array}$ \\
\hline Ownership of assets & $\begin{array}{l}\text { Rules for the acceptable use of information and } \\
\text { assets associated with information processing } \\
\text { facilities shall be identified, documented and } \\
\text { implemented. }\end{array}$ \\
\hline
\end{tabular}

\section{تقوم هذه المرحلة بتوقع المخاطرة بمحاولة تقدير المخاطرة بطريقتين:}

الأرجحية (Likelihood): هنا يكون احتمال أن تكون المخاطرة حقيقية.

• • • • • • • لعواقب (Consequence) : النتائج أو التبعات الناتجة عن المشاكل المتعلقة بالمخاطرة عند وقوعها.

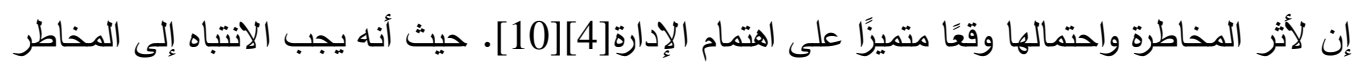

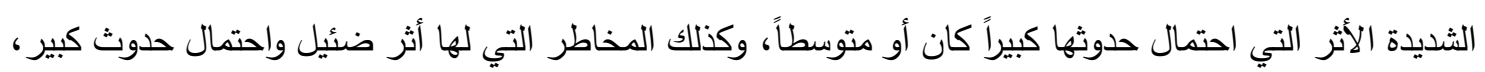

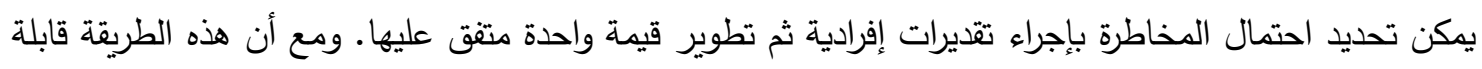

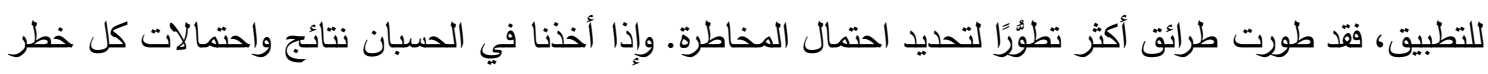
متضمنها مثل الجدول رقم (1)، يصبح من الممكن إعطاء الأولوية للأخطار الرئيسية والتي تحتاج إلى التحليل بطريقة أكثر تفصيلا. [10] نفحص، خلال تقييم المخاطرة، دقة التقديرات التي أجريت أثناء توقُّع المخاطرة، ونحاول ترتيب أولويات المخاطر التي كثِف عنها والبدء بالتفكير بطرائق لضبط أو تجنب المخاطر التي يحتمل حدوثها يتم

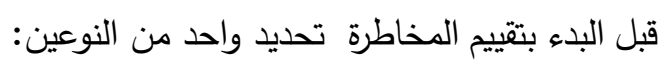

المستوى المرجعي للمخاطر (Risk Referent Level): يجب تعريف المستوى المرجعي للمخاطرة حتى

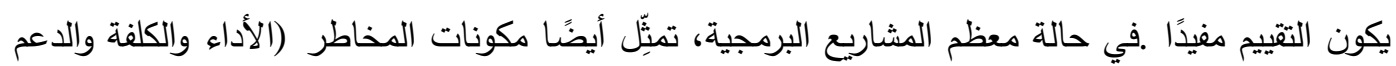

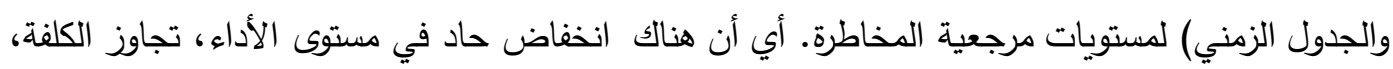

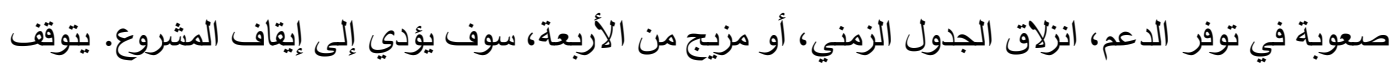

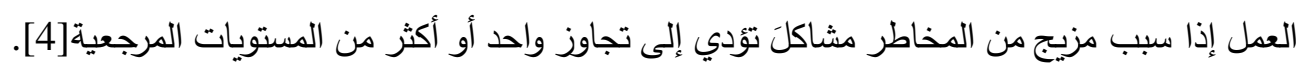
النقطة المرجعية (Reference Point): في سياق تحليل المخاطر البرمجية، يكون لمستوى المخاطرة نقطة

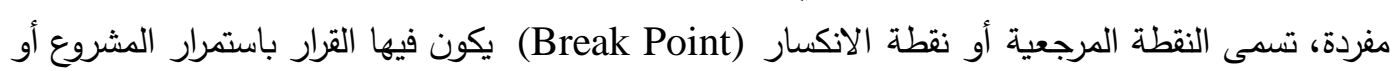

$$
\text { إلغائه (حين تكون المشاكل كبيرة). }
$$


يكون لقرارات الاستمرار أو الإنهاء عند النقطة المرجعية الوزن ذاته في بعض الوقت بل يكون في معظم

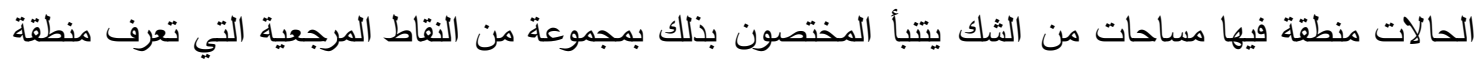

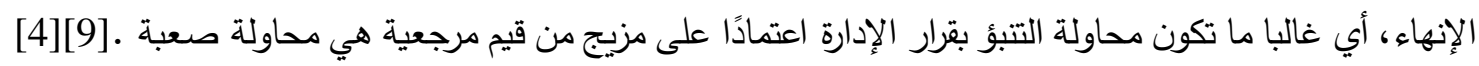

3.5 تخمين المخاطر :

إن الطريقة التي سنتبعها في تقدير أو تخمين المخاطر هي طريقة من الطرق الذكائية الخاصة بنظام

$$
\text { التضبيب الرقمي. }
$$

\section{6. الأنموذج الضبابي المستخدم:}

إن المجموعة الضبابية هي أصناف من العناصر مع درجة انتماء وان هذه المجموعات ميزت بدالة انتماء

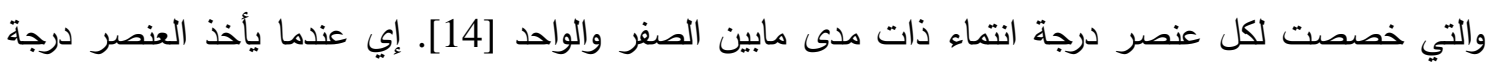
انتماء (1) فهذا يعني أن العنصر ينتمي بالتمام إلى المجموعات الضبابية، وعندما تكون درجة الانتماء (صفر) فهذا يعني إن العنصر لا ينتمي إلى المجموعة الضبابية، والدرجات الأخرى تتفاوت ما بين الصفر والواحد، عندما

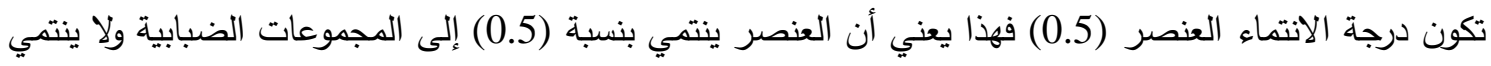

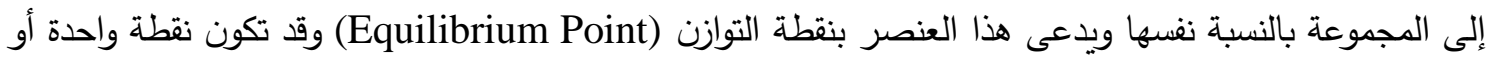
عدة نقاط. وعندما تكون درجة الانتماء (0.9) فهذا يعني أن العنصر ينتمي إلى المجموعة الضبابية بنسبة (0.9) ولا ينتمي إليها بنسبة (0.1) وهو أقرب إلى الانتماء من عدم الانتماء. أما المجموعة الاعتيادية "crisp set"

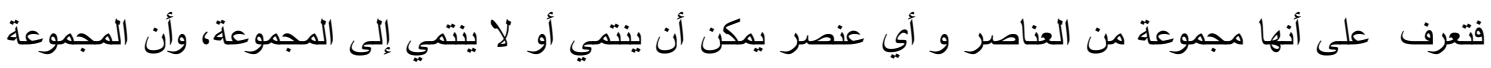

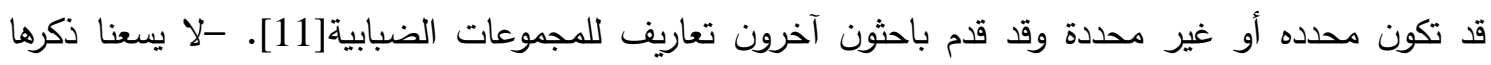
الآن -. إن اعتماد أنموذج التضبيب الذي يحاكي المتغيرات في أنموذجنا المستخدم هو الاعتماد على بيئة القرارات

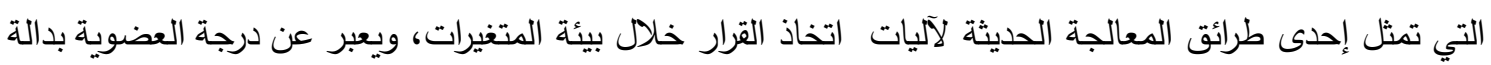
الانتماء MA(X) التي تمثل درجة انتماء العنصر من المتغير X إلى المجموعة الضبابية A وتكتب بالثكل الآتي: $\mu_{\mathrm{A}}: \mathrm{x} \rightarrow[0,1]$

$$
\mu_{A}(x)=\left\{\begin{array}{lll}
0 & \text { if } & \mathrm{x} \notin \mathrm{A} \\
1 & \text { if } & \mathrm{x} \in \mathrm{A}
\end{array}\right\}
$$

يتغير الانتماء للعناصر من العضوية التامة إلى عدم العضوية ، فهي إما أن تمتلك عضوية تامة، أو عضوية جزئية

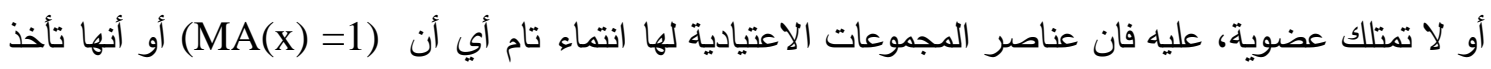

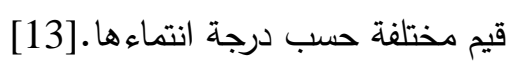

1.6 العدد المضبب:

عند العمل مع قيم مضببة وجعل الأداء الحسابي واضح، يجب استخدام مساحة كبيرة من التقييمات والتي

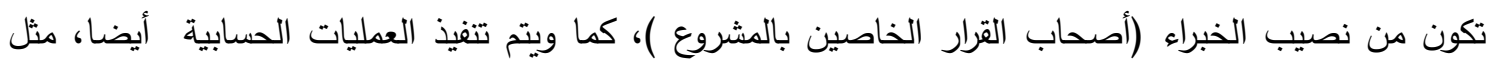

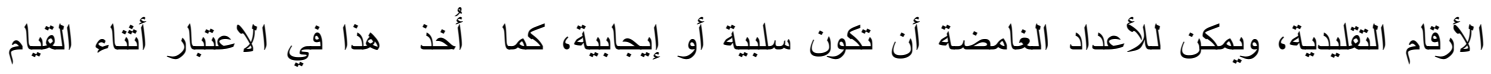

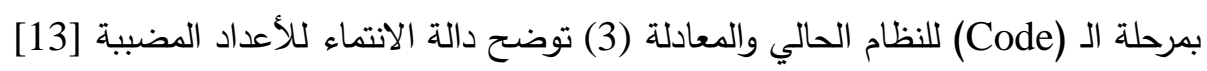




$$
\mu_{\tilde{A}}(x)= \begin{cases}\frac{x-p}{q-p}, & \text { if } p \leq x \leq q \\ \frac{r-x}{r-q}, & \text { if } q \leq x \leq r \\ 0, & \text { otherwise }\end{cases}
$$

إذ أن (p > r p قيم الحد الأدنى، المركز ، الحد الأعلى وتمثل فترة مغلقة لمدى الخطورة للسمة كما مبين في الثكل (3). ليس شرط وجوب البداية من الصفر حيث تكون قيم مختلفة[13].

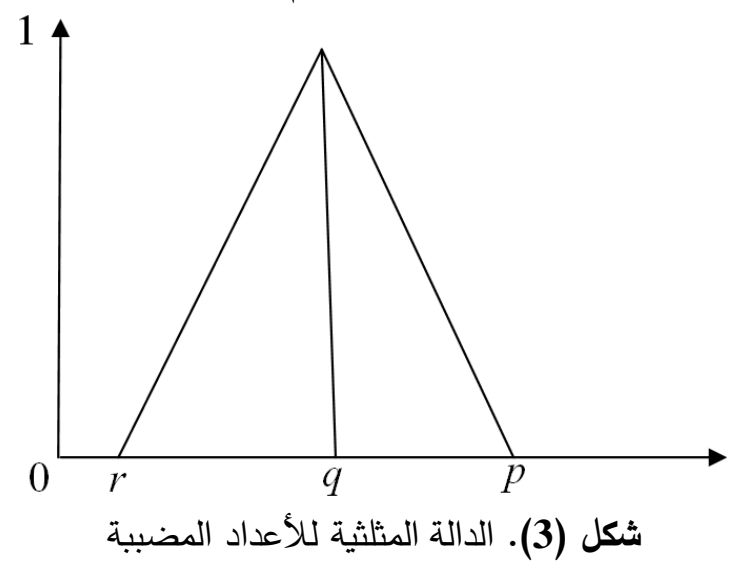

7. الأنموذج التحليلي المستخدم لنظام التقييم:

تم استخدام الأنموذج اللولبي من بين نماذج هندسة البرمجيات وذلك لوجود التفاصيل التي نحتاجها أثناء

مراحل التحليل.

\section{7 المرحلة الأولى ( تحليل السمات):}

باعتبار أن أول مراحل أنموذج العمليات المذكور سابقا (spiral model) هي مرحلة التحليل وهناك عدة

أنماط من طرق التحليل وهي حوالي 3 أنماط هي: مركز الدعم لتقنية البرمجيات Software STSC) و ومعهد هندسة البرمجيات Technology Support Center) (risk management studies by Dr. Barry Boehm) بتحليل سمات المخاطر للمشروع (Attributes) على دراسة إدارة المخاطر للدكتور باري بوهيم التي اعتمدت في مختلف المشاريع الاقتصادية في الدول المتطورة فيما يلي نذكر هذه

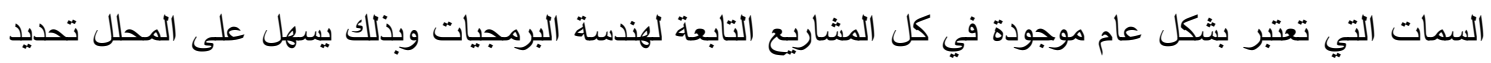
المخاطر الأساسية لأي نظام

م متطلبات النظام (System Requirements) O متعلقات الأشخاص (Personnel Collaborations)

م الجدولة والميزانية (Schedules and Budgets)

م تقنيات التطوير (Development Technology)

O المصادر الخارجية (External Resources)

[14].(performance) الأداء 


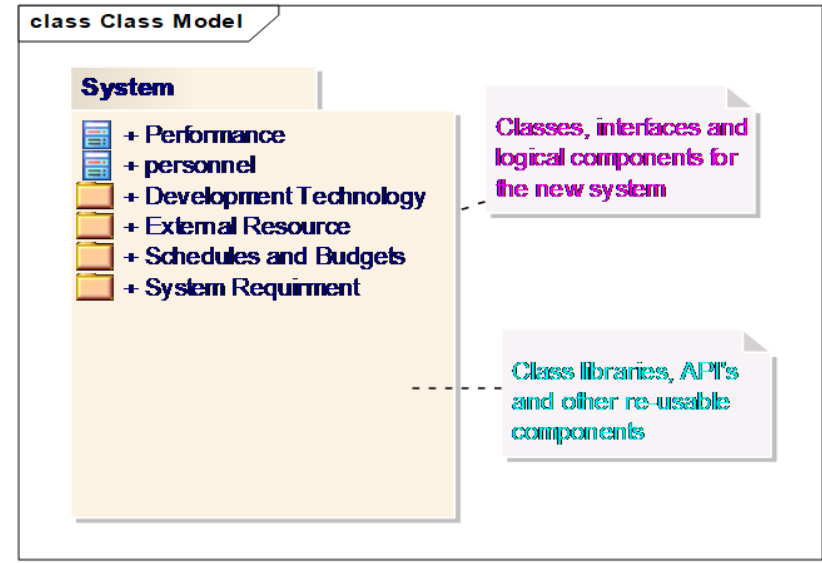

شكل (4). يوضح واجهة مخطط الصنف لأخطار بوهيم

O Development Technology, External Recourses, Schedule and Budgets, ( )

(System Requirement ) تحوي مجموعة من Risk) Items) وتسمى بالمجموعة (Package). O إن كل من (personnel, Performance) تعرف بالصنف (Class).

بعد ذلك نقوم بتحليل كل (Attribute) على حدة وتحديد نوعها ونوع التصنيف الذي ترجع إليه ويقوم

بهذه العملية محلل المخاطر (Risk Analyst).

وبعد عملية تحديد السمات يأتي دور صناع القرار وذلك بتحديد أوزان للسمات (Attributes) بحسب

درجة الخطر لها وحسب المتغيرات اللغوية المحددة سابقا حيث يكون أصحاب القرار أصحاب خبرة ومقيمين ماهرين ومتمرنين حيث كل سمة تحدد قيمتها وفق حدود أو فترات مغلقة لكل سمة حيث كل سمة تختلف عن الأخرى من ناحية أقصى حد علوي وأدنى حد سفلي. بعد ذلك يتم تطبيق المعادلة رقم (3) كما في الملحق رقم

فيما يلي الثكل (5) يوضح أنموذج مخطط حالة الاستخدام الكلي، الذي تكون بعد عملية تحليل السمات

الخاصة حسب تحليلات Barry Boehm.

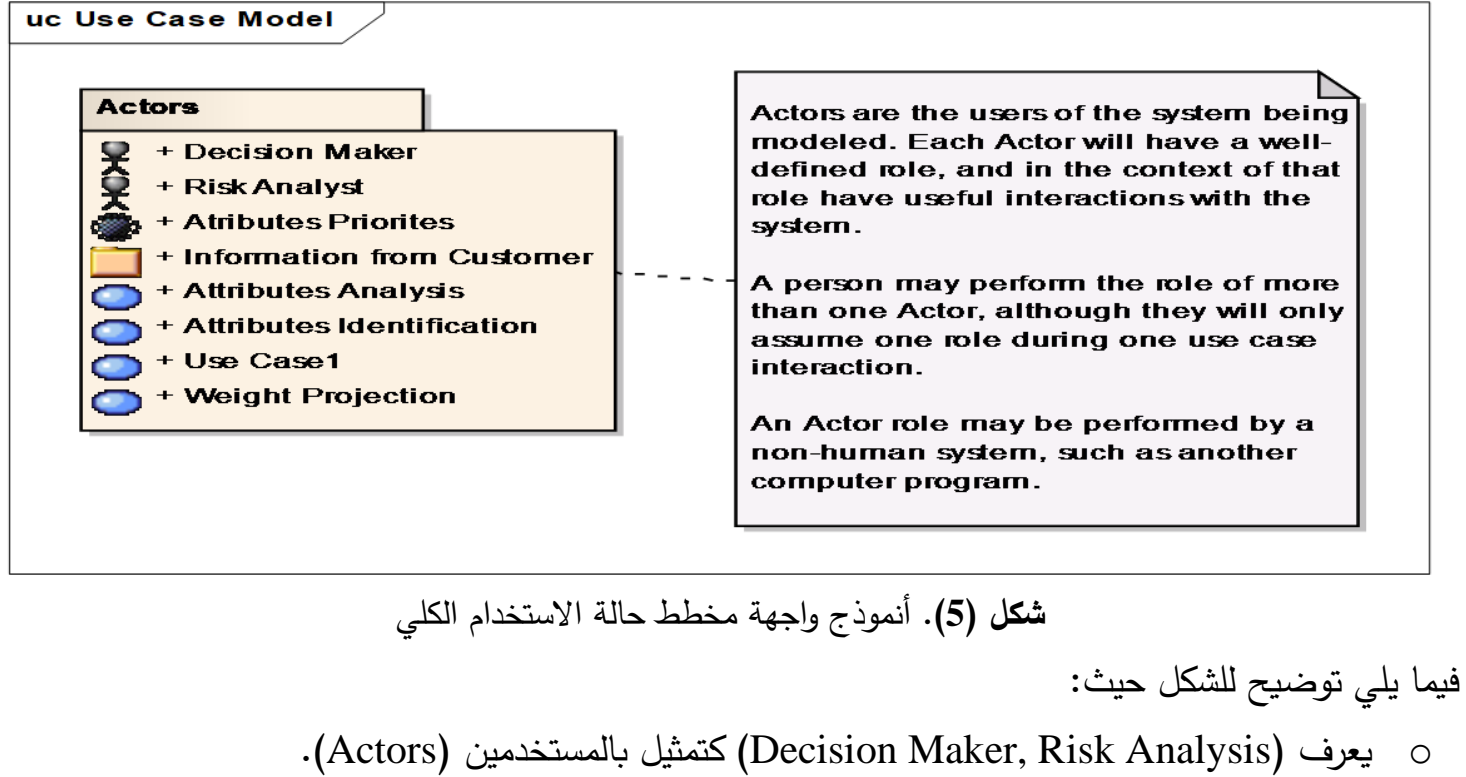


O يعرف (Attributes Priorities) بالارتباطات (Collaborations) المتعلقة بالسمات.

م يعرف (Information From Customer) بالمجموعة الخاصة بالسمات (Aackage).

م يعرف كل من (Attributes identification, Attributes Analysis, Weight Projection) بحالة بعرفة بات

الاستخدام.

بعد ذلك تتم معالجة الأوزان وذلك بتطبيق الدالة المثلثية للأعداد المضببة ( Triangular Fuzzy (Numbers حيث سينتج لنا لكل قيمة من الأوزان الخاصة بال (Attributes) أعداد مضببة تساعد في تقييم القيمة الكلية لنسبة المخاطر للمشروع.

2.7 المرحلة الثانية (تحليل عناصر الخطر):

وفقاً لأنموذج (Spiral Model) إن تحليل السمات في المرحلة السابقة تعتبر تحليلا أوليا، في هذه

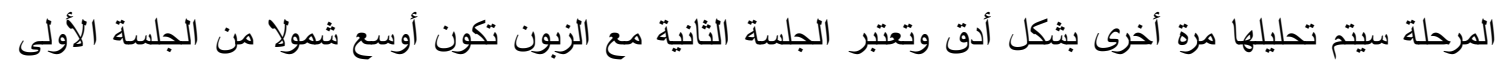
حيث سيظهر لنا ما يسمى بعناصر الخطر (Risk Items). تعرف (Risk Items) بأنها تفصيل لأنواع السمات (

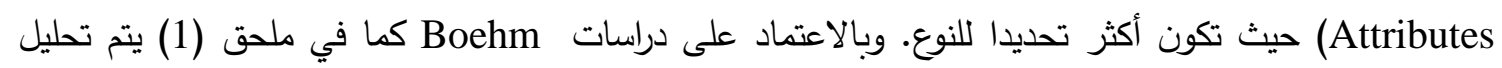
السمات Attributes إلى عناصر الخطر ( Risk Items) التي تعتبر التفصيل الدقيق لها.[4]

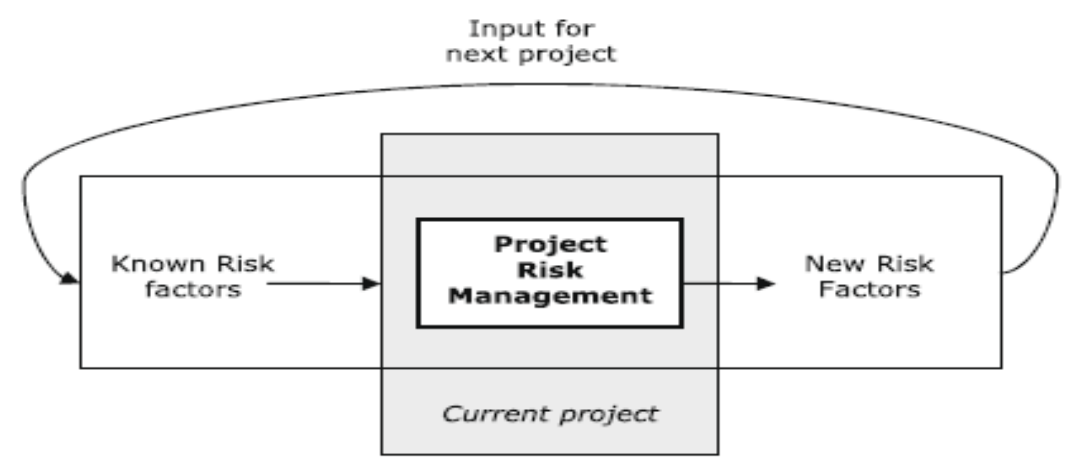

شكل (6). يوضح عملية التحليل التنصيلي

نلاحظ من الثكل (6) إن في كل جلسة حوارية مع الزبون تتتج لنا معلومات جديدة [4]. بعد ذلك يأتي دور مصنعو القرار في تتييم الوزن الخاص لكل (Risk Item). ومن ثم تطبيق القيم المقيمة على الدالة المثلثية للأعداد المضببة، سينتج ثلاث أعداد مضببة خاصة بكل عنصر من عناصر الخطر الخاصة بكل السمات التابعة للمشروع.

المرحلة الثالثة (تحليل درجة الخطورة):

في هذه المرحلة سيكون الاهتمام فقط على عناصر الخطر دون السمات لتحديد قيمة الخطورة لكل (Risk Item)

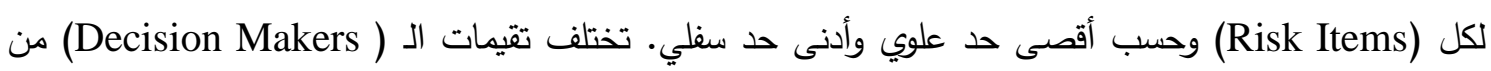
شخص إلى آخر كل حسب نظرته في التقييم ويحدث انه يتفقان على قيمة معينة ويحدث أيضا عدم الاتفاق بل والاختلاف يكون شاسعا بعض الأحيان. بعد ذلك سنقوم بتطبيق القيم المقيمة بالدالة المثلثية للأعداد المضببة. 
4.7 المرحلة الرابعة (تحليل درجة الأهمية):

نتبع في هذه المرحلة نفس خطوات المرحلة الثالثة بكل التفاصيل، لكن في هذه المرحلة سيتم تحديد درجة

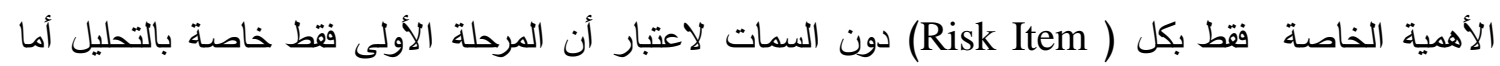

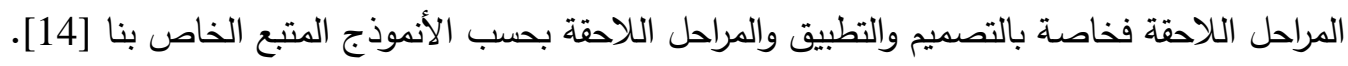

8. إجراء العمليات الخاصة بعملية التضبيب الرقمي:

Attributes, Risk item,Grad of Risk Grade of ) بعد مرحلة التحليل التي شملت كل من (Importance تبدأ العمليات الخاصة بالتضبيب الرقمي (Fuzzy Number) والتي تطبق على المعلومات

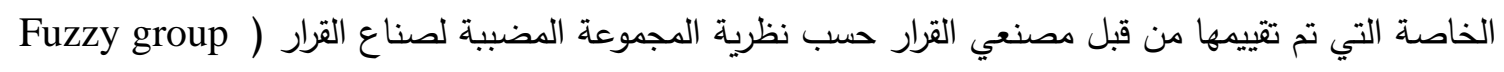
(Decision Makers

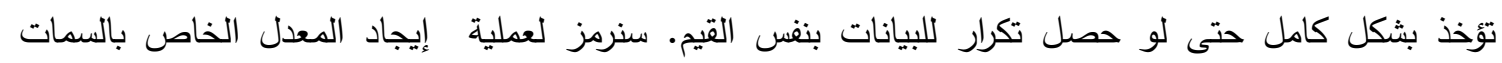
بالرمز A2 بأ Atributes) $A 2(h)=\frac{\sum_{i=1}^{2} D\left(\tilde{W}_{2(i, h)}\right)}{2}$

حيث أن:

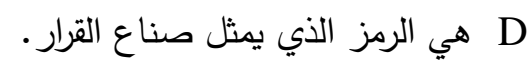

تمثل الوزن المقيم الخاص بالسمات. W2

تمثل عدد صناع القرار باعتبار أن لدينا اثثين من صناع القرار . تصنل

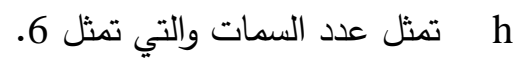

وبعد إيجاد المعدل الكلي الخاص بكل سمة لكل مصنعي القرار نقوم بإيجاد المعدل الكلي الخاص بكل عنصر

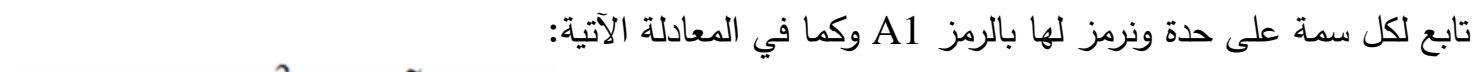
$A 1(h, k)=\frac{\sum_{i=1}^{2} D\left(\tilde{W}_{1(i, h, k)}\right)}{2}$

حيث أن:

D هي الرمز الذي يمثل صناع القرار D

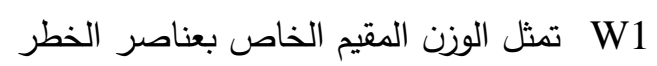
تمثل عدد صناع القرار باعتبار أن لدينا اثثين من صناع القطر القرار

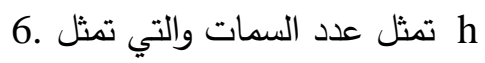
k تمثل رقم عنصر الخطر التابع لسمة معينة. بعد ذلك نقوم بإيجاد معدل درجة الخطر (average of grade of risk) لكل عنصر خطر فقط وليس السمات، $A R(h, k)=\frac{\sum_{i=1}^{2} D\left(\tilde{r}_{(i, h, k)}\right)}{2}$

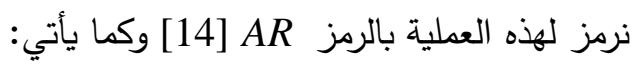

حيث أن: i تمثل عدد صناع القرار باعتبار أن لدينا اثثين من صناع القرار 


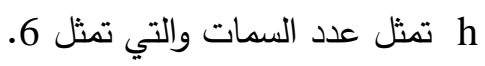

k تمثل رقم عنصر الخطر التابع لسمة معينة.

تمثل الوزن الخاص بدرجة الخطر الخاصة بعنصر الخطر تابع لسمة معينة. بعد ذلك نجد معدل درجة الأهمية لكل عنصر خطر من عناصر الخطر التابعة للسمات لكلا المقريين في

$$
A I(h, k)=\frac{\sum_{i=1}^{2} D\left(\tilde{i}_{(i, h, k)}\right)}{2}
$$

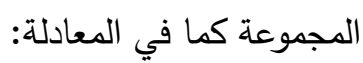

حيث أن:

i تمثل عدد صناع القرار باعتبار أن لدينا اثثين من صناع القرار

h تمثل عدد السمات والتي تمثل 6 و

k تمثل رقم عنصر الخطر التابع لسمة معينة.

تمثل الوزن الخاص بدرجة الخطر الخاصة بعنصر الخطر التابع لسمة معينة.

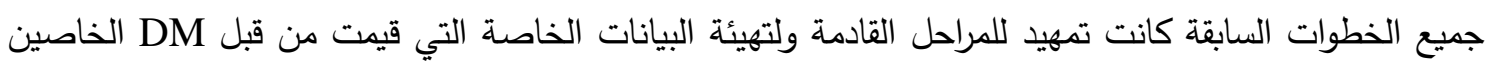
بالمشروع، حيث يؤخذ معدل نسبة الخطر الخاص بال Risk Item التابعة لا (Attribute) الواحد لكل واحدة

$$
G(h, k)=A R(h, k) \times A I(h, k)
$$
على حدة كما في المعادلة الآتية:

G: تمثل معدل نسبة الخطر الخاصة بال (Risk Items) الواحد بشكل منفرد. (grad of risk) تمثل درجة الخطورة : الخاصة بال (Risk Items) الواحد بشكل منفرد.

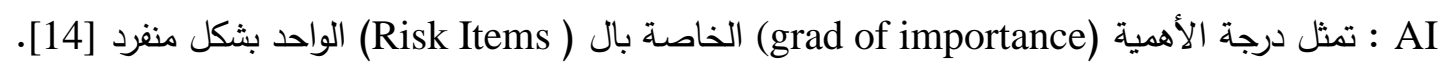
بعد هذه المرحلة سنحتاج إلى إيجاد القيمة المطلقة للأوزان الخاصة بالسمات أيضا بشكل منفرد كل سمة على حدة الإل الئل

$$
W\left(X_{h}\right)=\frac{A 2(h)}{\sum_{i=1}^{6} A 2(i)}
$$$$
\text { لكل سمة محددة كما في المعادلة رقم (6): }
$$

حيث أن:

Xh رقم تسلسل الوزن الخاص بالسمة (Attribute).

تمثل الوزان الخاص بالسمة الواحدة A2 تمثل تسلسل أرقام السمات

وبنفس الوقت نقوم بإيجاد القيمة المطلقة للأوزان الخاصة بكل عنصر خطر بحسب التسلسل الخاص ضمن السمة الواحدة حيث يعد الترتيب بالتسلسل مهم من حيث درجة الخطورة والأهمية. المعادلة التالية توضح هذه لوفئ العملية: $W\left(X_{h k}\right)=\frac{A 1(h, k)}{\sum_{i=1}^{n(h)} A 1(h, i)}$

W Xhk تمثل القيمة المطلقة للأوزان الخاصة بكل عنصر خطر بحسب التسلسل الخاص ضمن السمة الواحدة 
Al : تمثل الوزن الخاص بكل عنصر خطر

n(h)

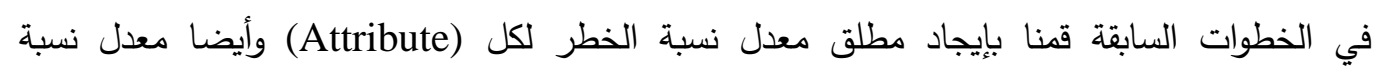

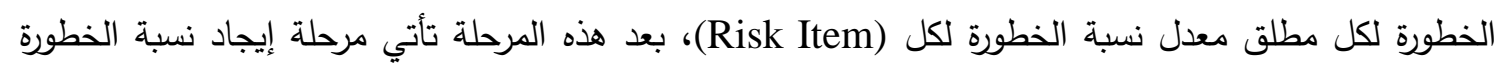

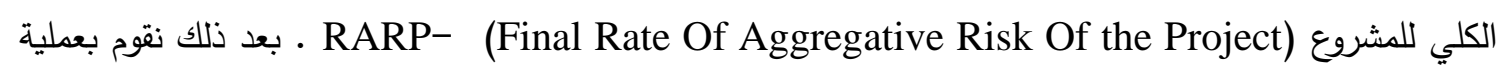

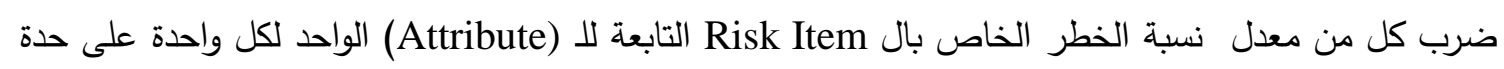

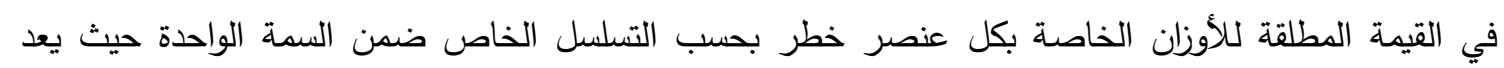

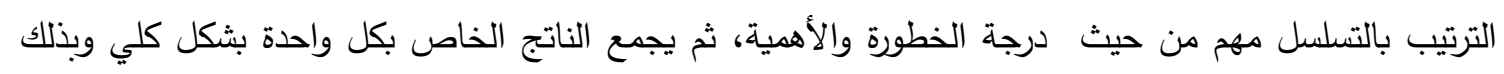

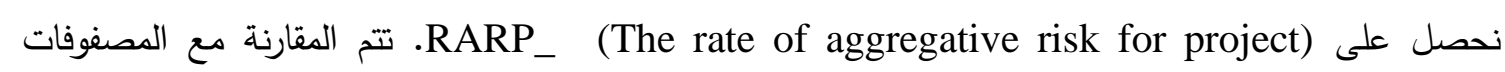
الخاصة بالمقاييس والمعايير الدولية مثل مصفوفة 3×3 و5×5.516]

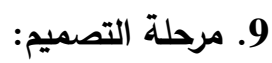

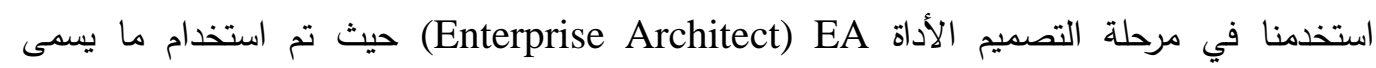

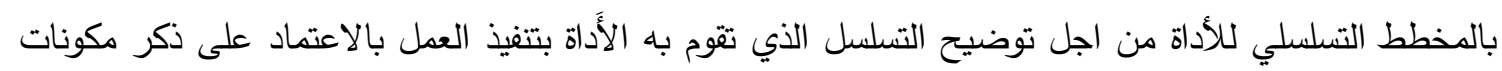

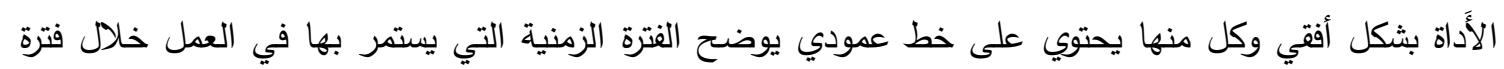

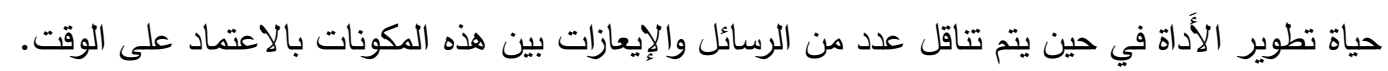

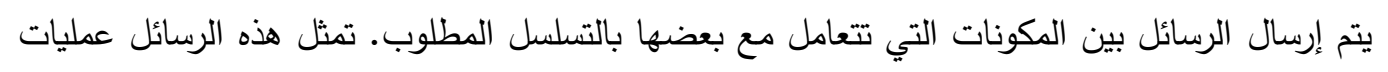

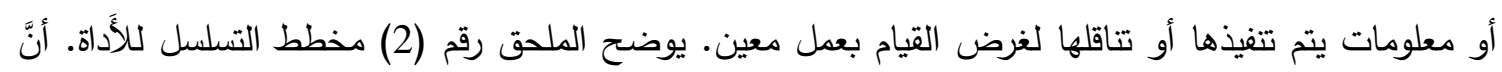

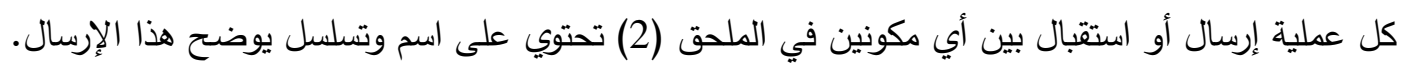
10. مرحلة كتابة البرنامج للنظام (مرحلة الترميز): تمت كتابة البرنامج باستخدام لغة (Express Edition Visual C\# 2010) وهي من لغات شركة

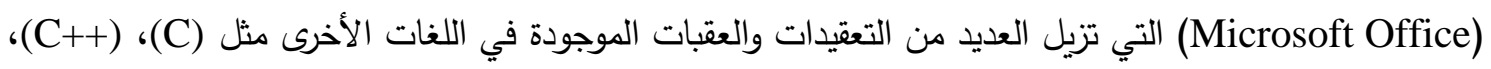

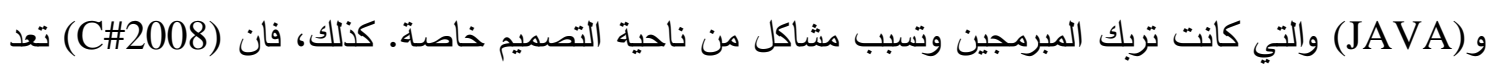

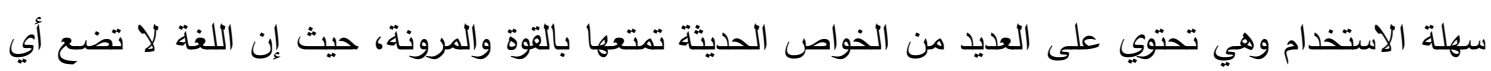

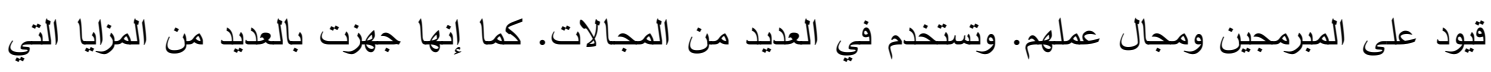

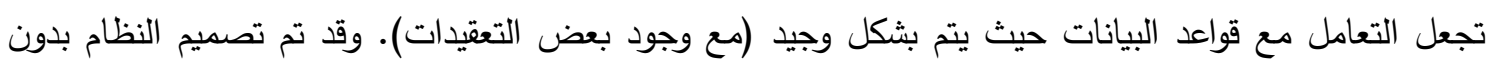

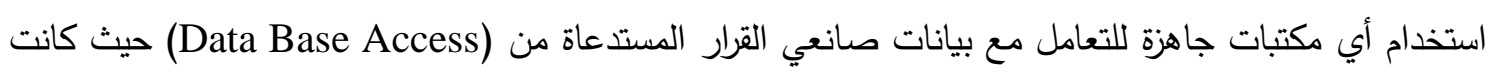

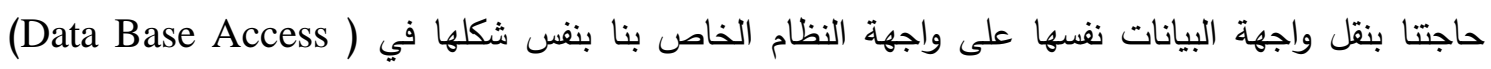
وكنلك تمت برمجة كل عملية من العمليات الرياضية.

11. - n مرحلة الاختبار:

تم اختبار النظام على نظامين من أنظمة البنوك الموجودة في العراق/ الموصل. البنك الأول هو بنك

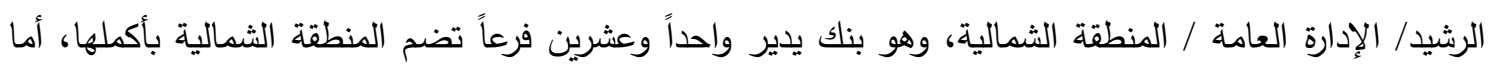

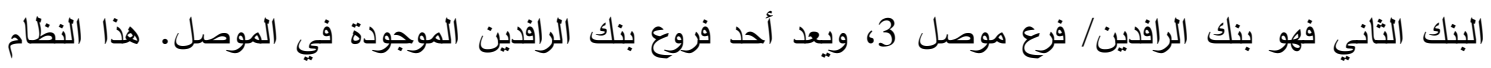


لبيانات ثلاثة مصنعي قرار بدرجات وظيفية معاون مدير عام مكتب مندوب/ بنك الرشيد / الإدارة العامة / المنطقة

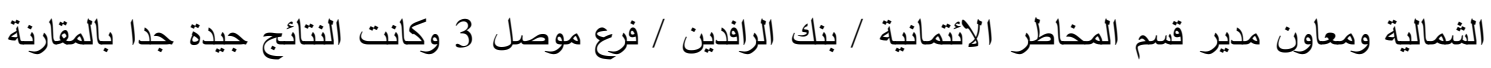
مع نسب البنوك للمخاطر الائتمانية التي عدت سابقا بمعدل 88\% بالأخذ بنظر الاعتبار النسب التي أخذت من صانعي القرار بشكل كلي.

12. - 12

1. التلازم بين النشاط الاقتصادي والمخاطرة يجعل كثف وتقييم المخاطر بشكل آني أمراً غير مدكن، لكن ذلك لا يعني بالضرورة عدم إمكانية التعامل معها وفق مجموعة من السياسات والاستراتيجيات التي تجعل آثارها

$$
\text { ونتائجها متحكم فيها إلى حد بعيد. }
$$

2. إن الأنموذج اللولبي هو من أتقن النماذج وأكثرها شمولا في إدارة المخاطر من النماذج الأخرى المتبعة في

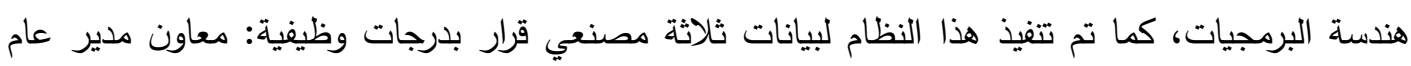

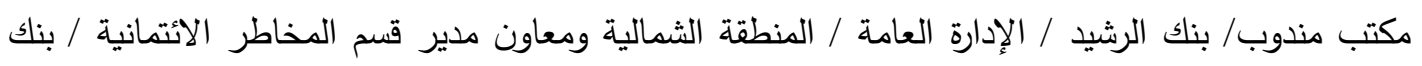

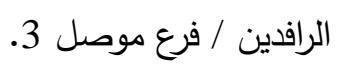

3. يعد الأنموذج الضبابي للأعداد المضببة أكثر دقة من العمليات الحسابية المتبعة في البنوك مثل قوانين كفاية

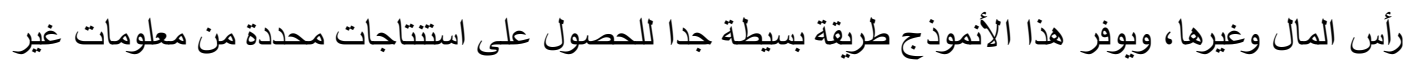

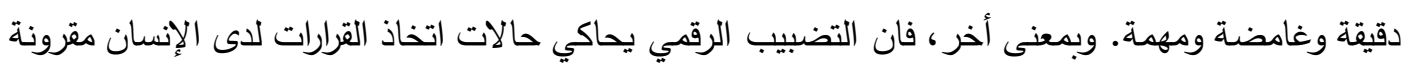
بالمحاولات لإيجاد حلول دقيقة من بيانات غير دقيقة أو تقرببية. حيث وجد أن الأنموذج الضبابي للأعداد المضببة مع استخدام برنامج EA بتمثيل مراحل التحليل والتصميم

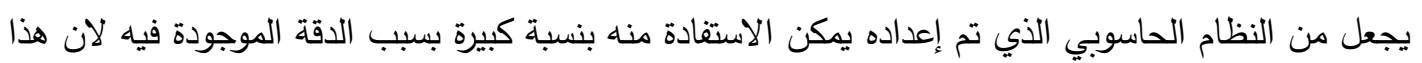

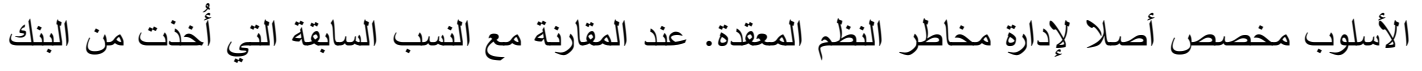

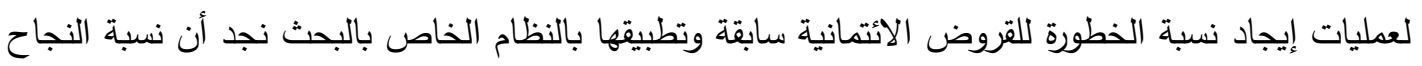
للقيم الناتجة من التطبيق المضبب تفوق النسب الناتجة من العمليات الخاصة بالبنك التقليدية حيث كانت النسبة 88\% من النسب بالأخذ بنظر الاعتبار النسب التي أخذت من صانعي القرار بشكل كلي. الجدول رقم

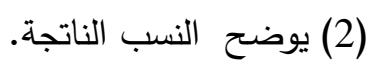


جدول (2). يوضح تباين النسب الكلية

\begin{tabular}{|c|c|c|}
\hline النسب الناتجة لاتقبيم & بالتضبية الناتجة التقيبيم & الطريقة المتبعة التيقيمي \\
\hline$\% 70$ & $\% 88-80$ & تقيبيم المخاطر الخاصة بينك الرشيد بوجود 6 Attribute 2 من صناع القرار \\
\hline$\% 60$ & $\% 86-77$ & و 1 صانيم المخاطر بيناك الراقدين 3 بوجود 6 attribute \\
\hline$\% 63$ & $\% 80-70$ & 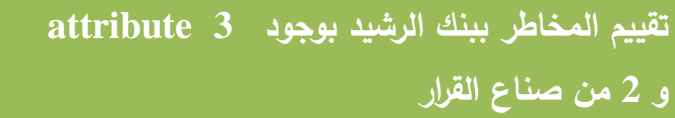 \\
\hline$\% 60$ & $\% 88-80$ & 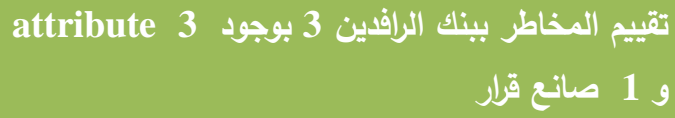 \\
\hline
\end{tabular}

1. يمكن تطوير العمل الحالي باتجاهات مختلف. ولعل من التوصيات الملحة هو تطوير النظام لمعالجة الأخطار

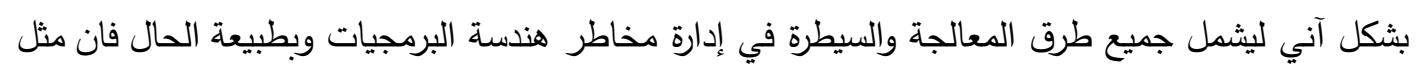
هذا المشروع يتطلب معلومات وتفاصيل إضافية تحتاج إلى جهود وتخصصات إضئل إضافية.

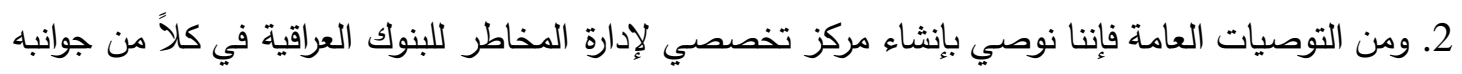

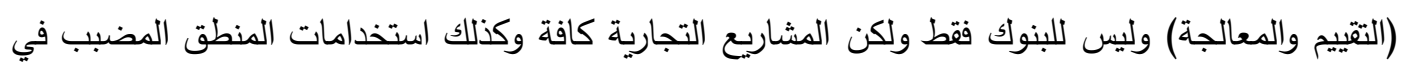
عمليات التحليل والسيطرة لدقته في التقييم. 


\section{المصادر}

[1] Mohamad, Jasni Zain Wan Maseri Wan Mohd Eyas El-Qawasmeh (Eds.) "Software Engineering and Computer Systems" Second International Conference, ICSECS 2011, Kuantan, Pahang, Malaysia, June 27-29, 2011 Proceedings, Part III

[2] Gillian Adens "The Role of Risk in a Modern Software Development" Process First Published: February 2004 ,Last Updated: January 2011 ,Copyright 20042010, Tassc Limited. All Rights Reserved.

[3] Karin van den Berg "Finding Open options An Open Source software evaluation model with a case study on Course Management Systems "Master Thesis Tilburg, August 2005

[4] Roger S. Pressmqn, Ph.D. "Software Engineering: Practitioner, Sa Pproachs, seven Edition .published by McGraw-Hill, a business unit of The McGraw-Hill Companies, Inc.c opyright @ 2010 by The McGraw-Hill Companies

[5] Gillian Adens, Robert Armstrong "Software Project Risks" First published: August 2001 Last Updated: January 2011 Copyright 2001-2009, Tassc Limited. All Rights Reserved.

[6] Pratap k.j .mohapatra "software Engineering", Copyright (C) 2010, New Age International (P) Ltd., Publishers Published by New Age International (P) Ltd., Publishers

[7] Jakub Miler, Janusz Górski, October -2001 "Implementing risk Management in software projects" Department of Applied Informatics Technical University of Poland

[8] Jonsas.Ekeroot, "Audio Software Developmet", 2007, Master Thesis, Lulea University of Technology

بلعزوز بن علي، جامعة الثلف، "استراتيجيات إدارة المخاطر في المعاملات المالية"، 2009، مجلة

$$
\text { الباحث. }
$$

[10] Jim Stevens, Alex Sprague "Risk Assessment in Software Development Projects, Contributors November 20, 2011. "Examining the Value of Risk Assessment and the Models of Risk in Software Development, Version 2.3.

فاضل عباس، الطائي ونجلاء، سعد ألثرابي، "نموذج الانحدار الذاتي المضبب مع التطبيق"، المجلة

$$
\text { العراقية للعلوم الإحصائية، } 2009 .
$$

[12] Alexia, Pacheco, Oldemar, Rodrguez, "Principal Components Analysis for Trapezoidal Fuzzy Numbers", Costarican Institute of Electricity.

[13] Lily Lin, and Huey-Ming Lee A Fuzzy Assessment Model, 2007, International Journal Of Computers, Issue 3, Volume, 2007.

[14] Jared, Bene, "Risk Management for Software Intensive Systems Common Risks" 2003, DOD Acquistion.

[15] Shyi-Ming Chen, "Fuzzy group decision making", 1997, Department of Electronic Engineering, National Taiwan University of Science and Technology, Taipei, Taiwan, Republic of China.

[16] Paul Barringer, P.E "Risk Matrix: Know when to accept the risk. Know when to reject the risk. Barringer \& Associates, Inc. 2006 
ملحق (1):

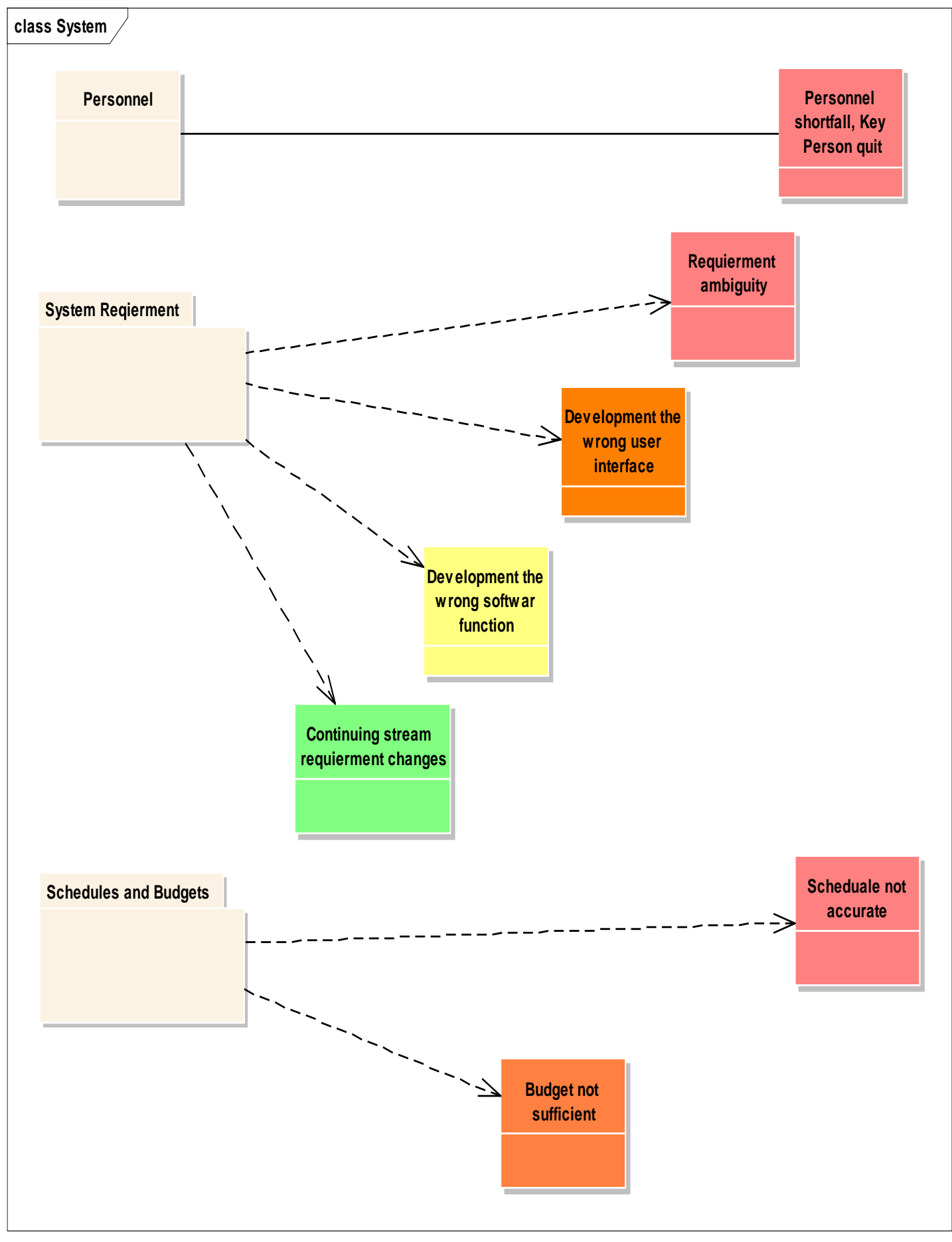

يوضح مخطط الصنف لتحليل المخاطر، عناصر الخطر، الأولوية 
بناء نظام برمجي بإتباع النموذج اللولبي لتقييم الخطر الإجمالي للمشاريع باستخدام نظرية التضبيب الرقصي

ملحق (2):

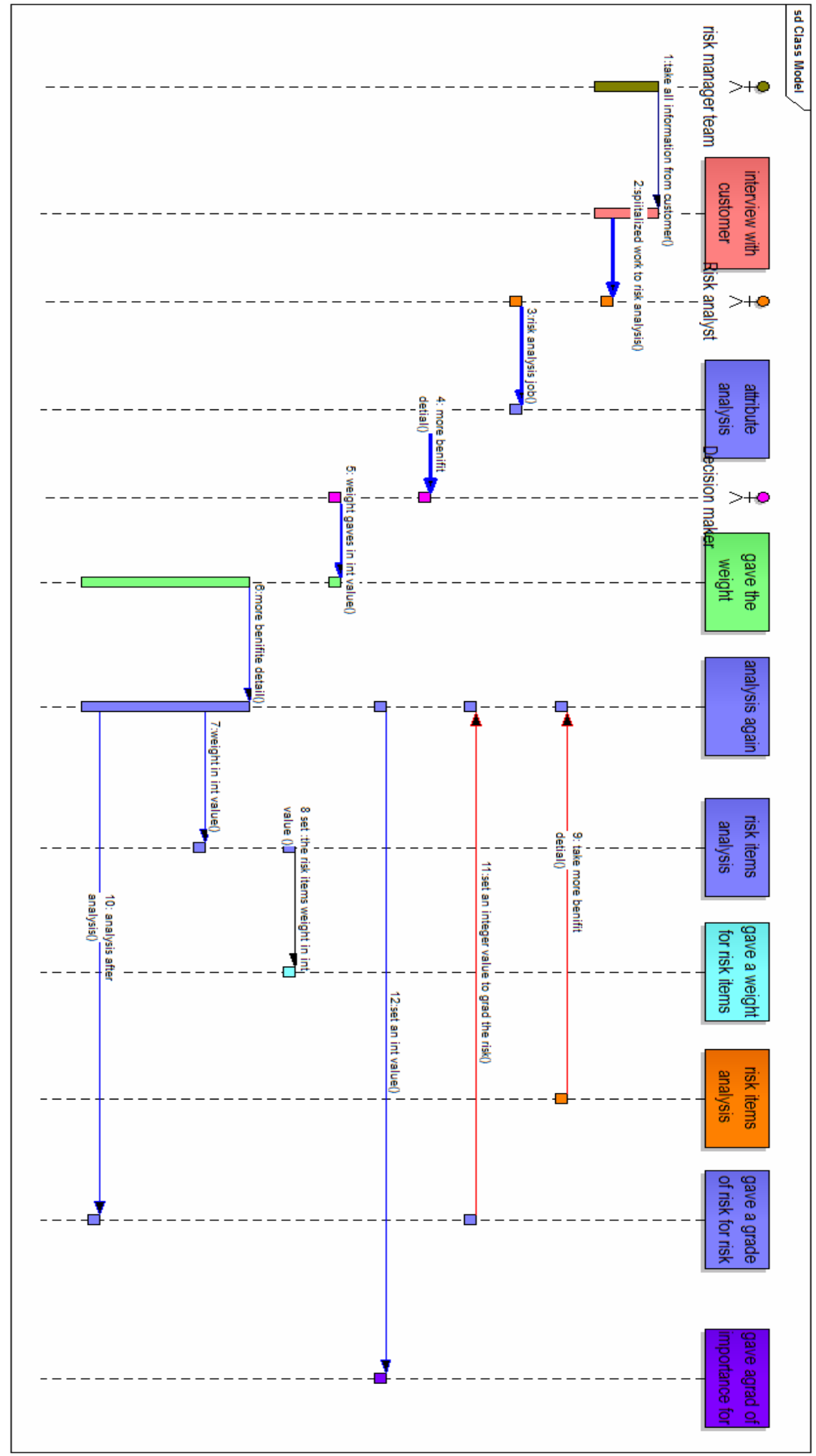

شكل (2). يوضح المخطط التسلسلي لتصميم النظام 
ملق (3)

$$
\text { مثال على حساب قيم السمات: }
$$

يتم وضع القيمة الخاصة بالسمة من قبل فريق العمل وكمثال على ذلك يهتم المحلل_ الذي يعتبر احد

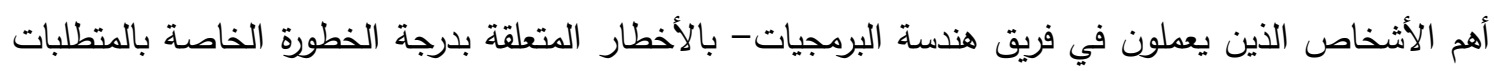
للنظام (والتي بدورها تحوي 4 عناصر خطر بعد تحليلها) وبعد ذلك يأتي دور صانع القرار رقم1 1 فيعطيها قيمة مثلا 60\% (فقط للسمة دون عناصر الخطر) فتؤخذ هذه القيمة وتتم معالجتها بالدالة المثلثية للأعداد المضببة التي

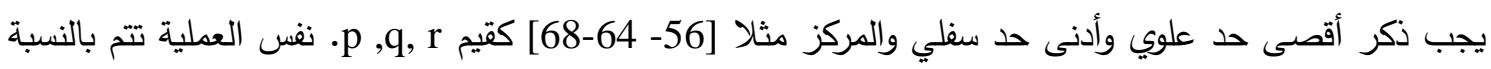

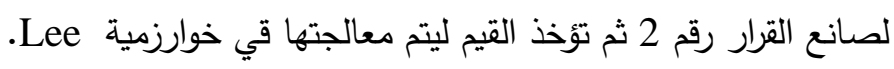

